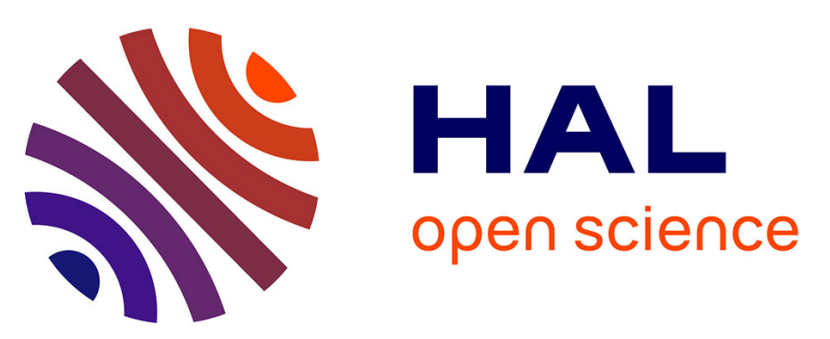

\title{
High-Density Bit Patterned Media: Magnetic Design and Recording Performance
}

M Grobis, Olav Hellwig, Thomas Hauet, Elizabeth Dobisz, T.R. Albrecht

\section{To cite this version:}

M Grobis, Olav Hellwig, Thomas Hauet, Elizabeth Dobisz, T.R. Albrecht. High-Density Bit Patterned Media: Magnetic Design and Recording Performance. IEEE Transactions on Magnetics, 2011, 47 (1), pp.6-10. 10.1109/TMAG.2010.2076798 . hal-01345374

\section{HAL Id: hal-01345374 \\ https://hal.science/hal-01345374}

Submitted on 18 Jul 2016

HAL is a multi-disciplinary open access archive for the deposit and dissemination of scientific research documents, whether they are published or not. The documents may come from teaching and research institutions in France or abroad, or from public or private research centers.
L'archive ouverte pluridisciplinaire HAL, est destinée au dépôt et à la diffusion de documents scientifiques de niveau recherche, publiés ou non, émanant des établissements d'enseignement et de recherche français ou étrangers, des laboratoires publics ou privés. 


\title{
High-Density Bit Patterned Media: Magnetic Design and Recording Performance
}

\author{
Michael K. Grobis ${ }^{1}$, Olav Hellwig ${ }^{1}$, Thomas Hauet ${ }^{1,2}$, Elizabeth Dobisz ${ }^{1}$, and Thomas R. Albrecht ${ }^{1}$ \\ ${ }^{1}$ Hitachi Global Storage Technologies, San Jose Research Center, San Jose, CA 95135 USA \\ ${ }^{2}$ CNRS-Nancy-Université-UPV-Metz, Vandoeuvre Les Nancy, France
}

\begin{abstract}
We examine the magnetic properties and recording performance of bit patterned exchange coupled composite (ECC) magnetic media at different bit and island aspect ratios. The $\mathrm{ECC}$ media consists of $\mathrm{Co} / \mathrm{Pd}$ and $\mathrm{Co} / \mathrm{Ni}$ multilayers whose coupling is controlled using $\mathrm{Pd}$ interlayers. We show that this multilayer system can be tuned to provide writeable media with a low switching field distribution for bit patterned magnetic recording. The recording performance of $100 \mathrm{~Gb} / \mathrm{in}^{2}$ media shows a sub $1 \mathrm{e}-4$ bit error rate floor and misregistration errors that are well-described by a simple error model.
\end{abstract}

\section{INTRODUCTION}

B IT PATTERNED MEDIA (BPM) is a promising approach for extending the densities in magnetic data storage beyond those achievable by conventional continuous perpendicular magnetic recording (PMR) [1]-[3]. In BPM recording, each bit is a lithographically defined single-domain magnetic island in contrast to PMR media in which a bit is composed of a disordered array of smaller magnetic grains. The larger effective magnetic volume of a BPM bit provides better thermal stability over conventional media and pushes the super-paramagnetic limit out to higher areal storage densities.

One critical challenge for BPM recording is achieving good write registration between the write process and the patterned bits [4]-[8]. A lack of synchronization between the write clock and the spatial location of the islands leads to on-track write errors, while cross-track misregistration leads to overwriting of adjacent tracks. A viable BPM recording system needs to have misregistration margins that allow for disturbances to occur while keeping the written-in error rate within levels that error-correction codes can handle. For a given head-media system, the misregistration margins depend in part on the switching field distribution (SFD) of the magnetic islands, head write field gradients, island size and position tolerances, island dimensions, and write head dimensions [4]-[6]. Narrow switching field distributions and high head field gradients give larger misregistration margins. An additional challenge for BPM recording is that write head design favors high bit aspect ratio (BAR) islands for achieving single track recording, whereas low BAR media is significantly easier to fabricate.

In this paper, we examine the magnetic performance of exchange coupled composite (ECC) bit patterned media with different bit and island aspect ratios [9]-[11]. The ECC media was tailored to give low SFDs and provide good writeability when using conventional magnetic recording heads. We examine the recording performance of the designed ECC media at $100 \mathrm{~Gb} / \mathrm{in}^{2}$ at BAR $=2.6$ and show how the error rate relates to fundamental properties of the recording system.

\section{EXChANGE COUPLED COMPOSITE MEDIA DESIGN}

To make ECC media, we used $\mathrm{Co} / \mathrm{Pd}$ and $\mathrm{Co} / \mathrm{Ni}$ multilayer structures as the composite components. Media with alternating layers of $\mathrm{Co}$ and $\mathrm{Pd}$ or $\mathrm{Ni}$ has magnetic anisotropy perpendicular to the layer planes. $\mathrm{Co} / \mathrm{Pd}$ has a higher anisotropy than $\mathrm{Co} / \mathrm{Ni}$ and we denote $\mathrm{Co} / \mathrm{Pd}$ as the hard layer and $\mathrm{Co} / \mathrm{Ni}$ as the soft layer. We tuned the exchange coupling between the composite components through the addition of Pd interlayers between the multilayer components. We show that such ECC-type structures allow the manipulation of the reversal mechanism in BPM and therefore controlling both the writeability (i.e., island coercivity) as well as SFD [11]. The BPM media was made by blanket deposition of the magnetic material onto pre-patterned Si wafer substrates. The substrates contained BPM at densities of $64-320 \mathrm{~Gb} / \mathrm{in}^{2}$ with a BAR and island aspect ratio (IAR) of one [11], [12].

In Fig. 1, we show an ECC-BPM system consisting of five $\mathrm{Co} / \mathrm{Pd}$ hard layer and two $\mathrm{Co} / \mathrm{Ni}$ soft layer repeats. Tuning the thickness of the Pd-interlayer in between the hard and the soft layer stacks from $9 \AA$ to $40 \AA$, we observe a uniform reversal of hard and soft layer up to a Pd-interlayer thickness of about $25 \AA$, while above that thickness hard and soft layer stacks reverse separately [Fig. 1(b)]. Decreasing the Pd interlayer thickness increases the coupling between the hard and soft layers, which increases the corresponding torque exerted by the soft layer on the hard layer in an applied field. The torque reduces the reversal field of the hard layer without compromising its thermal stability [9]-[11]. Below a critical Pd thickness, the torque starts to decrease as the large exchange coupling prevents appreciable tilting of the magnetization between the hard and soft layer. In our system, the maximal torque, and hence maximal reduction in reversal field, occurs at a $20 \AA$ Pd interlayer thickness [Fig. 1(c)]. Moreover, the magnetic SFD reveals a sharp minimum for the same Pd-interlayer thickness [Fig. 1(c)]. We conclude that ECC structures are highly favorable for BPM fabrication, since they provide a design point that allows very effective 


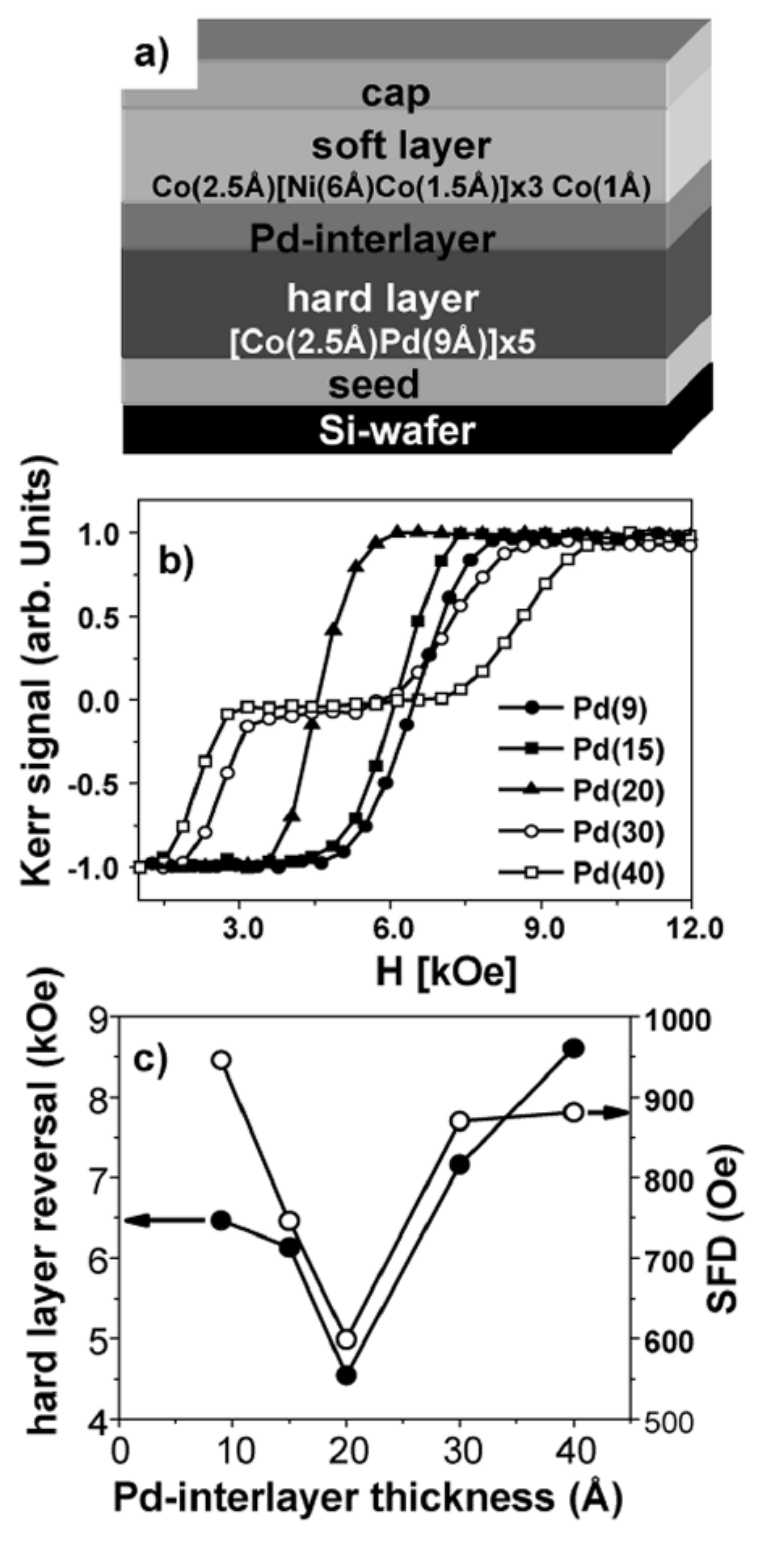

Fig. 1. ECC-type BPM consisting of five repeats $\mathrm{Co}(2.5 \mathrm{~A}) / \mathrm{Pd}(9 \mathrm{~A})$ as the hard layer and a $\mathrm{Co}(2.5 \mathrm{~A}) /[\mathrm{Ni}(6 \mathrm{~A}) / \mathrm{Co}(1.5 \mathrm{~A})] \times 3 / \mathrm{Co}(1 \mathrm{~A})$ structure as the soft layer. The Pd-interlayer thickness in between the top Co-film of the hard layer and the bottom Co-film of the soft layer was varied between $9 \mathrm{~A}$ and $40 \mathrm{~A}$ to tune the interlayer exchange coupling between the hard and the soft part. a) Illustration of the magnetic layer structure in our samples. b) Micromagneto optical Kerr effect (MOKE) remanent island reversal curves for various Pd interlayer thicknesses of ECC-type BPM at $180 \mathrm{Gbit} / \mathrm{in}^{2}$ ( $30 \mathrm{~nm}$ islands on $60 \mathrm{~nm}$ pitch, $\mathrm{BAR}=\mathrm{IAR}=1$ ). c) Hard layer reversal fields and SFD as a function of Pd-interlayer thickness for ECC-type BPM at $180 \mathrm{Gbit} / \mathrm{in}^{2}$.

lowering of the reversal field (writeability) while maintaining thermal stability and at the same time minimizing the magnetic SFD [11].

\section{BPM RECORDING MEDIA WITH BAR $>1$}

In order to perform recording experiments on these ECC-type BPM systems, we fabricated BPM on 2.5-in glass disks with a soft underlayer (SUL) and exchange break layer (EBL) structure below the BPM recording stack. The BPM was created by blanket deposition of magnetic media on top of etched SiNx pil- lars [12], [13]. To study the effects of island shapes on magnetic properties, we created several patterns with island aspect ratio (IAR) ranging from $1.6-3$ at a fixed density of $100 \mathrm{~Gb} / \mathrm{in}^{2}$ and fixed bit aspect ratio (BAR) of 2.6. The media consisted of 6 hard $\mathrm{Co} / \mathrm{Pd}$ repeats and $2 \mathrm{soft} \mathrm{Co} / \mathrm{Ni}$ repeats to produce a coercivity within the range of 6.5-7.5 kOe for recording purposes [14].

Fig. 2 shows magneto optical Kerr effect (MOKE) measurements and scanning electron microscopy (SEM) images of $100 \mathrm{~Gb} / \mathrm{in}^{2}$ over a range of island aspect ratios. While the $\mathrm{BAR}=\mathrm{IAR}=1$ structures in Fig. 2(a), (e) were fabricated on a Si-wafer, the higher BAR islands in Fig. 2(b)-(d), (f)-(h) were fabricated from SiNx deposited on an EBL/SUL on a 2.5 -in glass substrate. The variation in the island filling factor for different IAR affects the relative intensity of the trench and island signals in the micro-MOKE measurements. Lower island fill factors produce a lower island to trench signal ratio.

We observe a slight drop in the reversal field and an increase in the SFD of the islands on the patterned disks versus the $\mathrm{Si}$ substrates. This effect may be due to a slight increase in surface roughness for the glass substrates as compared to the $\mathrm{Si}$ wafers. These results are summarized in Fig. 3, where we show an illustration of the layer structure used in the BAR $>1$ experiments in Fig. 3(a) and compare ascending island reversal curves of the samples from Fig. 2 after normalizing to the island reversal signal amplitude.

In Fig. 3(c), we plot the total SFD versus IAR and find in addition to the above mentioned roughness effect, a significant increase in SFD when moving to higher IAR. As indicated by the SEM images in Fig. 2 the larger IAR islands also have larger island filling factor as well as a smaller spacing between island edges in cross track direction. Therefore, dipolar interactions between adjacent islands are larger for the larger IAR islands. Moreover, the oblong island geometry within the disk plane could contribute to a lowering of the reversal field [15]. Finally, the fabrication of smaller down-track dimensions and larger cross-track dimensions, which is necessary at higher IAR, yields larger relative variations in island width and island placement accuracy, which then contributes to a broader magnetic SFD as well.

\section{RECORDING EXPERIMENTS}

We examined the recording performance of the $100 \mathrm{~Gb} / \mathrm{in}^{2}$ media using a static tester in which the recording head is in contact with the disk [7], [16]. The bit cell was $50 \mathrm{~nm} \times 130$ $\mathrm{nm}$ with an island size of $40 \mathrm{~nm} \times 104 \mathrm{~nm}(\mathrm{BAR}=\mathrm{IAR}=$ 2.6). The ECC media contained 6 hard $\mathrm{Co} / \mathrm{Pd}$ repeats with 2 soft $\mathrm{Co} / \mathrm{Ni}$ repeats and a $5 \AA \mathrm{Pd}$ interlayer, as described in the previous section, but deposited on a different disk. The media had a coercivity of $6.5 \mathrm{kOe}$ and SFD of $1.0 \mathrm{kOe}$. We used a commercially available recording head with a mean write width well within the $130 \mathrm{~nm}$ track pitch of patterned islands. Fig. 4 demonstrates single track writing on this sample. We focused our study on measuring the on-track recording performance and in particular the effects of write phase misregistration on the bit error rate.

Write synchronization between the patterned islands and the switching of the write head field is paramount to achieving good 

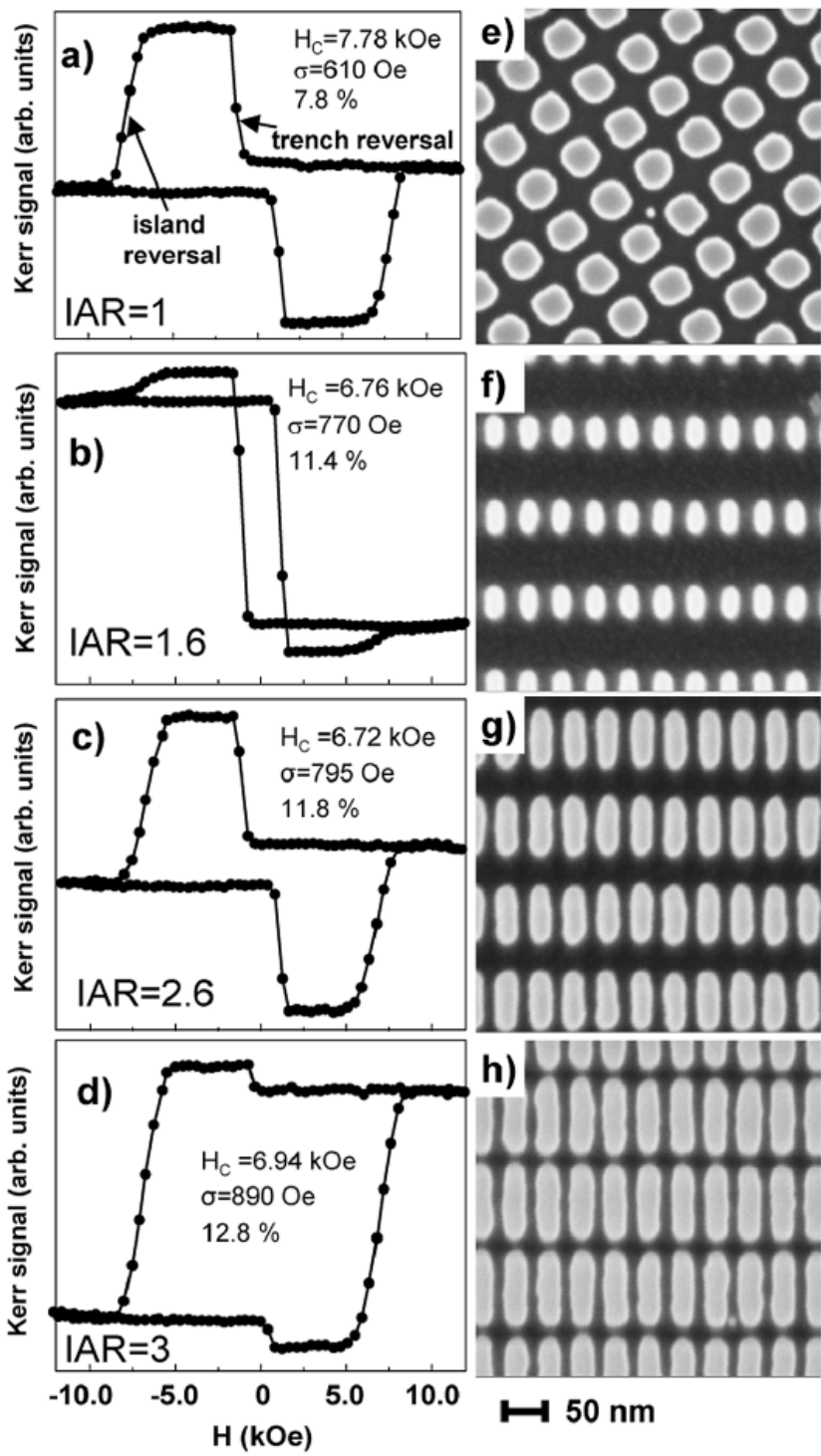

$\mapsto 50 \mathrm{~nm}$

Fig. 2. Micro-MOKE (a)-(d) and SEM data (e)-(h) of ECC-type BPM consisting of six repeats $\mathrm{Co}(2.8 \mathrm{~A}) / \mathrm{Pd}(9 \mathrm{~A})$ as the hard layer and a $\mathrm{Co}(2.8 \mathrm{~A}) /[\mathrm{Ni}(6 \mathrm{~A}) / \mathrm{Co}(1.5 \mathrm{~A})] \times 2 / \mathrm{Co}(1.3 \mathrm{~A})$ structure as the soft layer (see also Fig. 3(a)) as a function of IAR (IAR $=1$ (a), (e), IAR $=1.6$ (b), (f), $\mathrm{IAR}=2.6(\mathrm{c}),(\mathrm{g}), \mathrm{IAR}=3(\mathrm{~d}),(\mathrm{h})$. The IAR $=1$ structure was deposited on a $\mathrm{Si}$ wafer substrate with $\mathrm{BAR}=1$, while the $\mathrm{IAR}>1$ structures were deposited on glass substrates with an EBL/SUL structure. Here the BAR was kept constant at 2.6. For all 4 samples the Pd-interlayer thickness in between the top Co-film of the hard layer and the bottom Co-film of the soft layer was tuned to $14 \mathrm{~A}$ to optimize the coercivity. In the micro-MOKE measurements the magnetic trench reversal is visible at low fields $(\sim 1 \mathrm{kOe})$, while the island reversal occurs at around $7 \mathrm{kOe}$. The different sign of the trench and island reversal is purely optical in nature. Trenches and islands switch in the same direction during a field sweep.

recording performance. As Fig. 5 demonstrates, written-in errors can occur if the timing, or write phase, is not optimized. Fig. 5(a) shows high fidelity recording of a $\sim 2000$ bit pattern on a $60 \mathrm{~Gb} / \mathrm{in}^{2}$ sample with similar magnetic media to the one described earlier. In Fig. 5(b), the same pattern is recorded with the starting phase at each line advanced from the optimal phase and with a write frequency smaller than the optimal frequency.
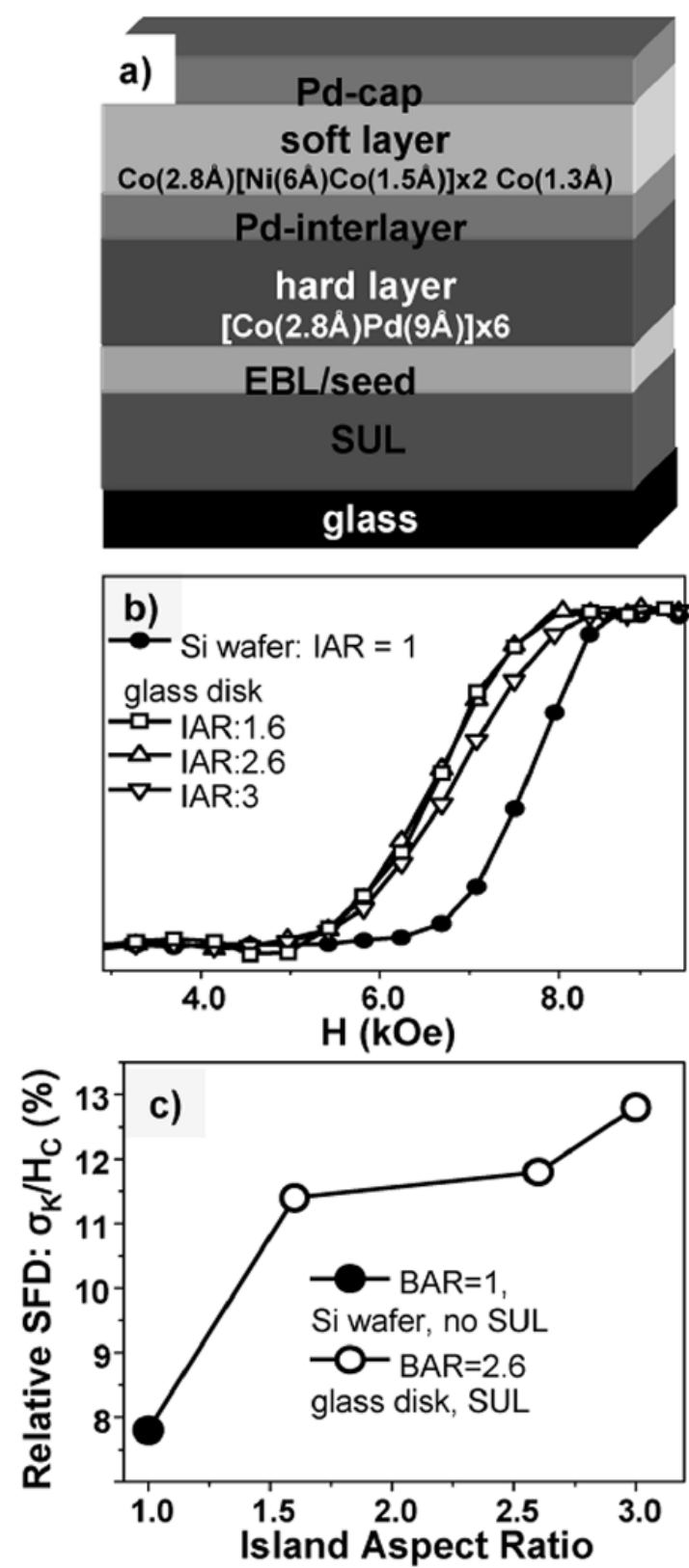

Fig. 3. ECC-type BPM consisting of six repeats $\mathrm{Co}(2.8 \mathrm{~A}) / \mathrm{Pd}(9 \mathrm{~A})$ as the hard layer and $\mathrm{Co}(2.8 \mathrm{~A}) /[\mathrm{Ni}(6 \mathrm{~A}) / \mathrm{Co}(1.5 \mathrm{~A})] \times 2 / \mathrm{Co}(1.3 \mathrm{~A})$ structure as the soft layer (a) as a function of IAR and BAR (see also Fig. 2). The IAR = 1 structure was deposited on a $\mathrm{Si}$ wafer substrate with a BAR $=1$, while the IAR $>1$ structures were deposited on glass substrates with an EBL/SUL structure. Here, the BAR was kept constant at 2.6. For all 4 samples the Pd-interlayer thickness in between the top Co-film of the hard layer and the bottom Co-film of the soft layer was tuned to $14 \mathrm{~A}$ to optimize the coercivity between 6.5 and 7 kOe on the glass substrate. In (b) we show the ascending branch of micro-MOKE hysteresis loops for various BAR/IAR values and in (c) we plot the relative SFD as a function of IAR.

Many errors occur at the start of each line, but diminish in number down track as the misclocking causes the write phase to decrease towards the optimal phase.

The effects of write phase on the bit error rate can be further quantified by measuring the written-in error rate as a function of 


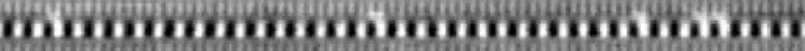

Fig. 4. $1 \mathrm{~T}$ tone written on a IAR $=2.6100 \mathrm{~Gb} / \mathrm{in}^{2}$ sample demonstrating single track writing. The media was prepped by dc magnetizing the disk. The white wisps in between the data tracks are trench domains. The scale bar is $1 \mu \mathrm{m}$.
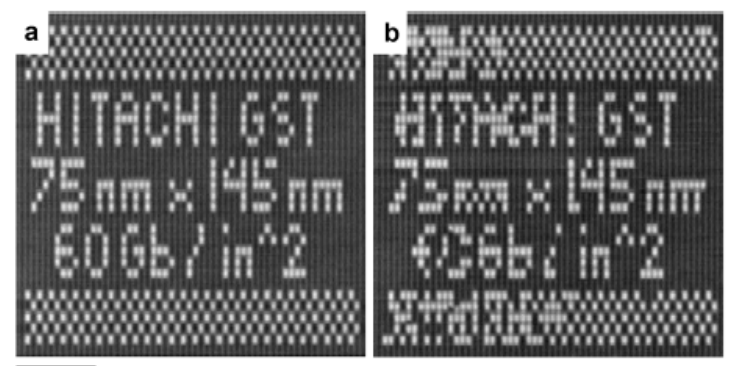

Fig. 5. Demonstration of the importance of write synchronization in BPM at $60 \mathrm{~Gb} / \mathrm{in}^{2}$. The scale bar is $1 \mu \mathrm{m}$. (a) Good write synchronization. (b) Incorrect start phase and incorrect frequency.

write phase. Fig. 6(a) shows the on-track bit error rate (OTER) versus write phase curve measured on the $100 \mathrm{~Gb} / \mathrm{in}^{2}$ sample with $\mathrm{IAR}=2.6$. The curve was compiled by writing random data and reading back the results using the static tester [17]. The write field was pulsed for $10 \mu$ s at each bit. Statistics were compiled for $\sim 15000$ islands with each track of islands written with 10 different write phases. The cross-track positioning error was around $\pm 5 \mathrm{~nm}$ and no adjacent track overwrite errors were observed. More details about the measurement technique are described elsewhere [18].

The bit error rate versus phase curve is well-described by a model that assumes that errors occur due to either overwriting of previously written islands or insufficient head fields at the effective write edge [4]-[6], [18]. The OTER can be defined as

$$
\operatorname{OTER}(\varphi)=\frac{1}{2}\left(P_{+}(\varphi)+P_{-}(\varphi)\right)
$$

Here, $\mathrm{P}_{+}$and $\mathrm{P}_{-}$are the probabilities for overwriting a previously written island and for having insufficient field to write the island at the write edge, respectively. $\varphi$ is the write phase normalized by the bit pitch $\mathrm{L}$. The prefactor of $1 / 2$ reflects the probability that the unintentional and desired write states are the same. For completeness, an additional constant term can be added inside the parentheses in (1) to account for merged or missing islands, super-paramagnetic islands, or islands which the head cannot switch. We do not include this term in our fits because the lack of errors for $|\varphi|<0.15$ in the OTER curve in Fig. 4(a) indicates that the number of such islands is beyond of the statistical resolution of our measurements. The lack of an error rate floor also shows the absence of readback errors in the measurement.

$\mathrm{P}+$ and $\mathrm{P}$ - depend on the switching field distributions, lithography jitter, write edge jitter, and write head profile. If we assume that the former three are uncorrelated Gaussian variables
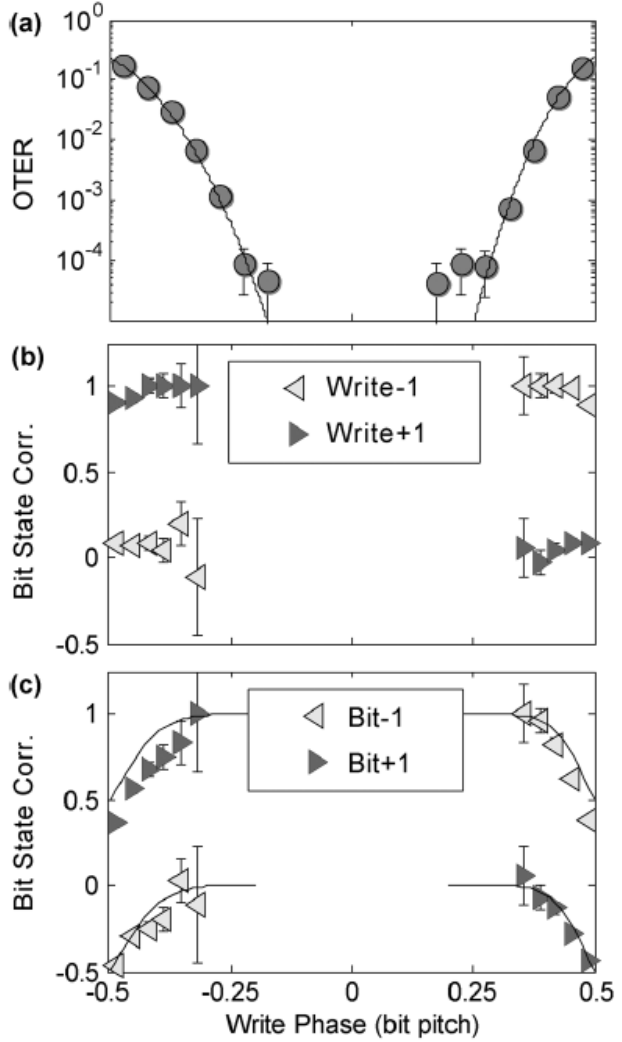

Fig. 6. Bit error rate versus write phase measurements at $100 \mathrm{~Gb} / \mathrm{in}^{2}$. (a) On track error rate (OTER) versus write phase. The black line is a fit to the data using equations (1)-(3). (b) Correlation between the written-in bit state of an erroneously written bit and the write states of the adjacent uptrack bit (Write-1) and downtrack bit (Write+1). (c) Correlation between the written-in bit state of an erroneously written bit and the written-in bit states of its uptrack (Bit-1) and downtrack (Bit+1) neighbors. The black line is fit to equation (4) and its counterparts for the other branches. We omit points in (b) and (c) for which the error bars are greater than \pm 0.5 .

and that the bit averaged write field is linear over the range of the switching field distribution, then

$$
\begin{aligned}
P_{ \pm}(\varphi) & =\frac{1}{2}\left(1 \mp \operatorname{erf}\left(-\left(\frac{\varphi \pm \frac{1}{2}}{\sigma_{ \pm}}\right)\right)\right) \\
\sigma_{ \pm} & =(1 / L) \sqrt{\left(\sigma_{K} / \partial H_{e f f} / \partial x\right)^{2}+\sigma_{L}^{2}+\sigma_{S}^{2}} .
\end{aligned}
$$

Here $\sigma_{\mathrm{K}}, \sigma_{\mathrm{L}}$, and $\sigma_{\mathrm{S}}$ are the standard deviations of the switching field distribution, bit position lithography jitter, and write timing jitter. The convention we use is that increasing $\varphi$ corresponds to increasing the down track position of the write head when the write field changes. $\partial \mathrm{H}_{\text {eff }} / \partial \mathrm{x}$ is the effective write field gradient. The two error processes can be governed by different net deviations that arise from different effective gradients or asymmetries in the various distributions.

The fit to (1)-(3) for the OTER versus phase curve is shown as the solid line in Fig. 6(a). The write phase is normalized by the $50 \mathrm{~nm}$ bit pitch and centered so that the maximum error rate occurs at $\varphi= \pm 0.5$. The fit matches the data nearly perfectly down to an OTER of 1e-4 at which point the measured OTER rises above the fit using the simple Gaussian error model. The best fit 
$\sigma_{+}$and $\sigma_{-}$were 0.079 and $0.060 \pm 0.002$, corresponding to a downtrack jitter in the effective bit position of $4.0 \mathrm{~nm}$ and 3.0 $\mathrm{nm}$, respectively. The measured lithography jitter was $0.8 \mathrm{~nm}$ and we estimate the head motion jitter to be less than $1 \mathrm{~nm}$. The measured $\sigma_{\mathrm{K}}$ of $1.0 \mathrm{kOe}$ gives an average effective write field gradient of $300 \mathrm{Oe} / \mathrm{nm}$ via (3). The asymmetry in $\sigma_{+}$and $\sigma_{-}$ most likely stems from nonuniformity of the effective head field gradient at fields within the switching field distribution of the media. $\sigma_{+}>\sigma_{-}$implies that the head field gradient is smaller at the switching fields of the softer islands than of the harder islands.

The error model described here can be verified further by examining the correlations between the written-in bit states and desired write states for bits that were written incorrectly. When an error occurs due to an overwriting of a previously written island the erroneous bit state should be the same as the write state of the successive bit. Likewise, if an error occurs due to insufficient field then the erroneous bit state should be the same as the write state on the previous bit since the write field extends several bits in the down track direction. Fig. 6(b) shows that these correlations indeed are observed for the erroneously written islands.

An analogous correlation is observed between the erroneous bit state and the bit state of neighboring bits, as shown in Fig. 6(c). While near $\varphi= \pm 0.3$, the error bit state to neighboring bit state correlations match the error bit state to neighboring write state correlations, the correlation values decrease by $\sim 0.5$ near the boundaries at $\varphi= \pm 0.5$. This slightly less intuitive result can be understood by noting that near $\varphi= \pm 0.5$ many write errors occur and the neighboring bit states may not be the same as the neighboring write states. To quantify this idea we examine the correlation between an erroneous bit state and the next downtrack bit state for $\varphi<0$. Here the dominant error mechanism is an overwriting of previously written bits and governed by $\mathrm{P}_{+}$in (2). If a bit has been erroneously written then its correlation to the next bit state depends on whether that bit was overwritten or not. Taking these two probabilities into account, we find that the erroneous bit state to next bit state correlation function should obey

$$
E C_{\mathrm{bit}+1}(\varphi<0)=1-P_{+}(\varphi) .
$$

A similar formula can be found for the other branches in Fig. 6(c). The solid black line in Fig. 6(c) shows the fit to the data using the set of equations related to (4). The fit obeys the data very well, though with some deviation seen most clearly in the upper branches. The deviation stems from uncertainty in assigning the write phase when writing near $\varphi= \pm 0.5$. Write errors near these points could be assigned to either boundary, which mixes the statistics between the corresponding branches.

\section{CONCLUSION}

ECC media employing $\mathrm{Co} / \mathrm{Pd}$ and $\mathrm{Co} / \mathrm{Ni}$ multilayers provides a flexible framework for tuning the magnetic properties of BPM for recording applications. The observed recording performance demonstrates the feasibility of single track recording at a sub $1 \mathrm{e}-4$ bit error rate at $100 \mathrm{~Gb} / \mathrm{in}^{2}$ with ample misregistration margins. Higher density recording has already been demonstrated with this type of media [18] and further progress can be made by optimizing the growth conditions and media stack composition in order to improve the SFD of the media and the match to the field profile of the recording heads.

\section{ACKNOWLEDGMENT}

The authors thank M. Schabes, G. Zeltzer, and B. Wilson for fruitful discussions and technical help.

\section{REFERENCES}

[1] B. D. Terris and T. Thomson, "Nanofabricated and self-assembled magnetic structures as data storage media," J. Phys. D-Appl. Phys., vol. 38, pp. R199-R222, Jun. 21, 2005.

[2] T. R. Albrecht et al., "Bit-patterned magnetic recording: Nanoscale magnetic islands for data storage," in Nanoscale Magnetic Materials and Applications, J. P. Liu, Ed. et al. Dordrecht: Springer, 2009, pp. 237-274.

[3] B.C. Stipe et al., "Magnetic recording at $1.5 \mathrm{~Pb} \mathrm{~m}-2$ using an integrated plasmonic antenna," Nature Photon., vol. 4, pp. 484 488, 2010.

[4] H. J. Richter et al., "Recording on bit-patterned media at densities of 1 Tb/in ${ }^{2}$ and beyond," IEEE Trans. Magn., vol. 42, pp. 2255-2260, Oct. 2006.

[5] H. J. Richter et al., "Recording potential of bit-patterned media," Appl. Phys. Lett., vol. 88, p. 222512, May 29, 2006.

[6] M. E. Schabes, "Micromagnetic simulations for terabit/in² head/media systems," J. Magn. Magn. Mater., vol. 320, pp. 2880-2884, Nov. 2008.

[7] M. Albrecht et al., "Recording performance of high-density patterned perpendicular magnetic media," Appl. Phys. Lett., vol. 81, pp. 2875-2877, Oct. 7, 2002.

[8] A. Moser et al., "Off-track margin in bit patterned media," Appl. Phys. Lett., vol. 91, p. 162502, Oct. 15, 2007.

[9] D. Suess et al., "Exchange spring media for perpendicular recording," Appl. Phys. Lett., vol. 87, p. 012504, Jul. 4, 2005.

[10] D. Suess et al., "Exchange-coupled perpendicular media," J. Magn. Magn. Mater., vol. 321, pp. 545-554, Mar. 2009.

[11] T. Hauet et al., "Role of reversal incoherency in reducing switching field and switching field distribution of exchange coupled composite bit patterned media," Appl. Phys. Lett., vol. 95, p. 262504, Dec. 28, 2009.

[12] O. Hellwig et al., "Coercivity tuning in $\mathrm{Co} / \mathrm{Pd}$ multilayer based bit patterned media," Appl. Phys. Lett., vol. 95, p. 232505, Dec. 7, 2009.

[13] S. Landis et al., "Domain structure of magnetic layers deposited on patterned silicon," Appl. Phys. Lett., vol. 75, pp. 2473-2475, Oct. 18, 1999.

[14] Typical recording media has $\mathrm{Hc} \sim 4.5 \mathrm{kOe}$. The Hc of the BPM we use for recording is higher to account for its higher Stoner-Wohlfarth exponent, which governs the dependence of $\mathrm{Hc}$ on field angle.

[15] J. Kalezhi et al., "Dependence of switching fields on island shape in bit patterned media," IEEE Trans. Magn., vol. 45, pp. 3531-3534, Oct. 2009.

[16] A. Moser et al., "Dynamic coercivity measurements in thin film recording media using a contact write/read tester," J. Appl. Phys., vol. 85, pp. 5018-5020, Apr. 15, 1999.

[17] The data values were determined from the readback waveform using a software read channel. Though, as Figs. 4 and 5 demonstrate, the data values at could have been deduced using threshold detection alone.

[18] M. Grobis et al., "Measurements of the write error rate in bit patterned magnetic recording at 100-320 Gb/in" ${ }^{2}, "$ Appl. Phys. Lett., vol. 96, p. 052509, Feb. 1, 2010. 